\title{
Dialyse und Nierentransplantation
}

\author{
Werner Riegel, Bernd Krüger
}

\section{Betablocker und Spironolacton}

Betablocker I Die positive Wirkung von Betablockern für Dialysepatienten war bereits Thema in unserem Beitrag in DMW 23/2014. Interessant ist nun, dass die Dialysierbarkeit der Betablocker eine signifikante Bedeutung hat. Dies zeigten Weir et al. [1] in einer bevölkerungsbasierten retrospektiven Kohortenstudie bei über 66-jährigen Patienten unter LangzeitHämodialyse, bei denen eine Betablockertherapie initiiert worden war: Eine Therapie mit hoch-dialysierbaren Betablockern (Atenolol, Acebutolol, Metoprolol) war mit einer signifikant höheren 180-Tage-Sterblichkeit assoziiert (relatives Risiko 1,4 [1,1-1,8]; $\mathrm{p}<0,01$ ) als die Therapie mit niedrig-dialysierbaren Betablockern (Bisoprolol, Propranolol).

Spironolacton | Seit vielen Jahren ist der positive Effekt von Spironolacton auf die Herzinsuffizienz bekannt. Die linksventrikuläre Hypertrophie wird zudem günstig beeinflusst. Auch in der Behandlung der arteriellen Hypertonie spielt Spironolacton eine wichtige Rolle, sobald der Verdacht auf einen Hyperaldosteronismus besteht. Nun ist diese Behandlung aber auch mit dem Risiko einer Hyperkaliämie behaftet, insbesondere wenn eine Verschlechterung der Nierenfunktion eintritt. Diese Gefahr besteht nicht mehr, wenn die Restdiurese bei Hämodialyse gering ist oder eine Peritonealdialyse (PD) durchgeführt wird. Bei PD kommt es eher zur einer Hypokaliämie. So bildet sie eine günstige Konstellation zum Einsatz von Spironolacton [2]. Die Gabe von $25 \mathrm{mg}$ Spironolacton für 2 Jahre führte bei 158 PDPatienten zu einer signifikanten Verbesserung des Verlaufs von linksventrikulärer Funktion und Masse, ohne vermehrte Hyperkaliämie. Letztlich könnten sowohl Herzinsuffizienz als auch das Peritoneum davon profitieren. Zhan et al. [3] zeigten in einer experimentellen Studie den positiven Effekt von Spironolacton auf Inflammation und Fibrose des Peritoneums bei Peritonealdialyse. Die Dicke des Peritoneums und die Entgiftungsfunktion bleiben davon unberührt. In einer weiteren Studie [4] waren bei 18 Patienten Kollagenbildung und Ablagerung von CD20Zellen gehemmt.

\section{Was ist neu?}

- Betablocker und Spironolacton: Betablocker mit hoher Dialysierbarkeit gingen in einer Studie bei Langzeit-Hämodialyse mit einer höheren Mortalität einher als solcher mit niedriger Dialysierbarkeit. Spironolacton hatte bei Patienten unter Peritonealdialyse positive Effekte.

- Gewicht bei Hämodialyse: Bei kleinen Patienten mit hohem BodyMass-Index zeigte sich unter Hämodialyse ein besseres Überleben als bei großen Patienten mit gleichem Gewicht.

- Antikoagulation: Patienten, die unter Vitamin-K-Antagonisten ausreichend antikoaguliert sind, brauchen möglicherweise zusätzlich kein Heparin. In einer Studie bei Dialysepatienten stiegen die Spiegel des löslichen VEGF-Rezeptors nach Heparin-Gabe stark an; dies könnte die Vasodilatation beeinträchtigen. Zu neuen oralen Antikoagulanzien bei Dialysepatienten sind noch nicht ausreichend Daten verfügbar.

- Peritonealdialyse (PD): Bei Patienten mit Lupus erythematodes war die PD trotz Immunsuppression erfolgreich. Auch nach Herz-Lungentransplantation hatte diese Dialyseform keine Nachteile. Eine perkutane endoskopische Gastrostomie ist eine relative Kontraindikation der PD.

- Spendernieren mit akutem Nierenversagen: Nieren von Spendern mit aktuem Nierenversagen haben im ersten Jahr nach Transplantation keine schlechteren Funktionsraten als Standardnieren mit normaler Funktion.

- Resistance-Index (RI) nach Nierentransplantation: Der RI war bei einem Cut-off von 0,80 nicht mit der Organfunktion sowie akuten oder chronischen Läsionen im Transplantat assoziiert. Vielmehr zeigte sich eine Assoziation mit dem Empfängeralter sowie hämodynamischen Faktoren. Möglicherweise korreliert der RI mit der Mortalität nach Nierentransplantation.
Klinische Relevanz
Bei hypertensiven Dialysepatienten könnten Betablocker mit niedriger Dialysierbarkeit Vorteile haben; dies muss aber weiter untersucht werden. Spironolacton könnte bei nicht ausreichend kontrollierter Hyper- tonie, linksventrikulärer Hypertrophie, Herzinsuffizienz und Peritonealdialyse häufiger eingesetzt werden. Allerdings muss auf eine Hyperkaliämie geachtet werden.

\section{Gewicht bei Hämodialyse}

Patienten mit hohem BMI haben bessere Überlebensraten bei der Hämodialyse - vorausgesetzt, diese Patienten sind klein [5]. Der Grund dafür liegt möglicherweise darin, dass sie eine niedrigere Muskelmasse haben als große Menschen mit gleichem Gewicht. Somit erhält die dicke und kleine Person eine höhere Dialysedosis in Relation zur vorhandenen Muskelmasse. Dicke Patienten sind deshalb dick, weil sie einen besseren Appetit 
haben, oder vielleicht weil sie gesünder sind, weniger Entzündung oder weniger Katabolismus haben. Gerade bei sehr gut genährten Patienten besteht aber auch das Problem der Hyperphosphatämie. Die Studie besticht durch eine hohe Fallzahl von 117644 Patienten, die in einem 5-Jahres-Zeitraum prospektiv untersucht wurden [5]. Bemerkenswerterweise geht die zunehmende Körpergröße ebenso wie das niedrige Körpergewicht mit erhöhter Sterblichkeit beim Dialysepatienten einher.

\section{Klinische Relevanz}

Die Körpergröße ist ein neu entdeckter Risikofaktor für die Sterblichkeit bei Dialysepatienten. Eine Gewichtsabnahme sollten Dialysepatienten in aller Regel vermeiden.

\section{Antikoagulation}

Heparin plus orale Antikoagulation? | Die Antikoagulation unter Hämodialyse ist ein wichtiges Thema. das immer wieder Fragen aufwirft. Krummel et al. [6] untersuchten in einer prospektiven Crossover-Studie 40 Dialyse-Sitzungen bei $10 \mathrm{~Pa}-$ tienten, die mit Vitamin-K-Antagonisten antikoaguliert waren, Die Sitzungen erfolgten mit zwei verschiedenen Membranen (Polysulfon oder Heparin-beschichtetes Acrylonitril) sowie mit und ohne Enoxaparin. Endpunkte waren die klinische Einschätzung der Blutgerinnung sowie verschiedene Gerinnungsparameter. Die Heparingabe beeinflusste die Ergebnisse nicht, und das Ergebnis war auch unabhängig von der eingesetzten Membran. Somit erscheint eine zusätzliche Heparingabe nicht notwendig

Löslicher VEGF-Rerzeptor I Die lösliche Form des Vascular-Endothelial-Growth-Factor-1-Rezeptors sFlt1 wird von Endothelzellen, Monozyten und plazentaren Zytotrophoblasten sezerniert und bindet VEGF. Es gibt Hinweise, dass die sFlt1-Spiegel mit kardiovaskulären Ereignissen korrelieren, wenn eine Niereninsuffizienz vorliegt. Lavanine et al. [7] zeigten, dass bei 48 Dialysepatienten die sFlt-1-Spiegel nach Heparin-Gabe dramatisch anstiegen. Bereits nach einer Minute stiegen die Spiegel von 102 pg/ml ausgehend und erreichten das Maximum von $2551 \mathrm{pg} / \mathrm{ml}$ im Mittel nach 15 Minuten. DiMarco et al. [8] spekulieren, dass ein sFlt1-Anstieg während der Dialyse kurzfristig zu einer Verminderung der Vasodilatation durch VEGF-Antagonismus führen kann. Die Verminderung der Myokarddurchblutung ist eine Folge. Auch die Nierengefäße sind betroffen. Albuminurie und direkte Gefäßschädigungen sind die Folge, die letztlich den Verlust der Nierenrestfunktion hervorrufen können. Heparin greift bei Dialysebehandlung durch Freisetzung von sFlt 1 in einen komplexen Regelkreis von Gefäß- und Wachstumsfaktoren eingreift. Die Konsequenzen sind noch nicht absehbar.
NOAK I Ob die neueren Antikoagulanzien (NOAK) eine Verbesserung dieser Situation bringen, ist noch offen. Bei moderatem bis schwerem chronischen Nierenversagen scheint Apixaban in Bezug auf Blutungsereignisse relativ sicher. Aktuell hat die FDA sogar bei Dialysepatienten allein auf der Basis der pharmakokinetischen Daten eine Dosierungsempfehlung gegeben [9].

\section{Klinische Relevanz}

Bei hämodialysierten Patienten unter Phenprocoumon sollte die zusätzliche Heparin-Gabe überdacht werden. Neuere Antikoagulanzien (NOAK) müssen zum jetzigen Zeitpunkt noch mit großer Vorsicht eingesetzt werden.

\section{Peritonealdialyse}

Immunsuppression | Insbesondere, wenn eine Immunsuppression nötig ist, besteht die Befürchtung, dass Patienten mit Peritonealdialyse stärker gefährdet sind. Bei systemischem Lupus erythematodes waren die Ergebnisse jedoch sehr günstig [10]. Auch nach Herz-Lungentransplantation hat die Peritonealdialyse (PD) keine Nachteile [11]. Im Gegenteil: Bei Hämodialysepatienten wurden höhere Hospitalisationsraten registriert. Infektionen der Lunge und Probleme mit dem Gefäßzugang waren dafür verantwortlich. Hinsichtlich Dialyseassoziierter Infektionen (Peritonitiden und Bakteriämien) unterschieden sich Patienten unter Peritonealdialyse und Hämodialyse nicht signifikant.

\section{Perkutane endoskopische Gastrostomie (PEG) I} Ein interessanter Aspekt ist die gleichzeitige Durchführung von Peritonealdialyse und Ernährung über eine PEG-Sonde. Die Frage nach dieser Behandlung stellt sich bei dialysepflichtigen Patienten mit Heimbeatmung. Die Versorgung in Heimen mit Intensivversorgung scheint möglich, ist aber im ambulanten Dialysesetting problematisch. Da das Peritonitis-Risiko bei PEG erhöht ist, bildet sie eine relative Kontraindikation für die Peritonealdialyse. Eine PEG unter laufender PD ist ebenfalls mit einem hohen Risiko assoziiert. Erforderlich sind eine Antibiotika- und antifungale Prophylaxe sowie eine 2-3-tägige Pause der Peritonealdialysebehandlung. Umgekehrt ist es leichter: Sobald eine PEG eingeheilt ist, kann die Peritonealdialyse erfolgen [12].

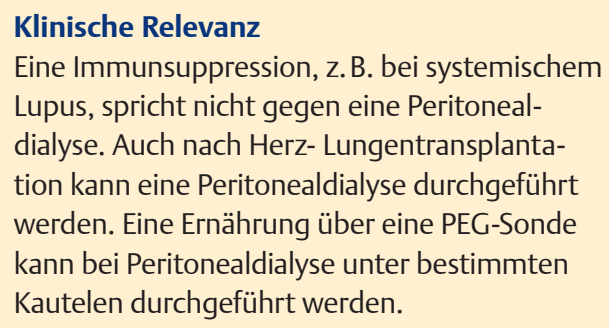




\section{Spendernieren mit akutem Nierenversagen}

In den vergangenen Jahren ist die Spendebereitschaft in Deutschland weiter gesunken - und dies, obwohl sie schon zuvor deutlich unter der in anderen Ländern wie z.B. Spanien lag. Daher gab es in den letzten Jahren verstärkte Bemühungen, den Spenderpool zu erweitern. So wurde untersucht, ob hirntote Patienten, die im Rahmen des Intensivaufenthaltes ein akutes Nierenversagen (ANV) erleiden, zur Gewinnung von Spendernieren mit akzeptablen Langzeitfunktionsraten in Frage kommen. In den letzten Monaten finden sich verstärkt retrospektive Fallserien, die eine erfolgreiche Transplantation von Spendernieren mit einem ANV beschreiben.

Daten aus den USA I Die US-Arbeitsgruppe um Farney [13] berichtete über Erfahrungen von 84 Transplantationen, bei denen die Spenderniere ein ANV aufwies; bei Aufnahme hatte das Serumkreatinin mit $1,25 \mathrm{mg} / \mathrm{dl}$ im Normbereich gelegen und war vor Explantation signifikant auf $3,22 \mathrm{mg} / \mathrm{dl}$ angestiegen. Dabei fanden sich keine Unterschiede im 5-Jahres-Organüberleben im Vergleich zu einer Kontrollgruppe ( $n=283)$, in der „normal“ funktionierende Nieren transplantiert worden waren. Interessanterweise ließ sich in der Gruppe, die eine ANV-Niere erhielt, nicht der bekannte negative Effekt einer verzögerten Funktion („delayed graft function"; DGF) auf das Organüberleben nachweisen. Allerdings unterscheiden sich mehrere Parameter von der Realitität in Deutschland:

- Spenderalter ca. 36 Jahre

- Maschinenperfusion in mehr als 50\%

- Kaltischämiezeit von ca. 25 Stunden

Daten aus Deutschland I Dass die Ergebnisse dennoch auch auf deutsche Verhältnisse übertragbar sind, zeigen zwei Studien:

- In einer Fall-Kontrollstudie [14] der eigenen Arbeitsgruppe aus Mannheim wurden $33 \mathrm{~Pa}-$ tienten, die eine Niere mit einem ANV transplantiert bekommen hatten (Kreatinin bei Entnahme 2,41 mg/dl), 65 Patienten mit Transplantation einer „Standardniere“, d. h. einer normalen Nierenfunktion bei Entnahme, gegenübergestellt. Die Funktionsaufnahme der transplantierten Nieren, gemessen am Serumkreatinin, war in der ANV-Gruppe verzögert, erreichte aber bereits nach 2 Wochen eine vergleichbare Funktion wie in der Kontrollgruppe. Auch wir stellten eine erhöhte DGF-Rate fest (42 vs. 27\%), die sich aber weder auf das Patienten- noch auf das Transplantatüberleben nach einem Jahr auswirkte.

- Bestätigt werden die Ergebnisse durch Daten der Gruppe um Jacobi [15] aus Erlangen. In die retrospektive Studie wurden alle Patienten eingeschlossen, die von 2008-2014 eine Leichenniere erhalten hatten. Von den 382 eingeschlossenen Patienten erhielten 174 eine Niere mit erweiterten Spenderkriterien, davon 63 die Niere eines Donors, der im Laufe des stationären Aufenthalts ein akutes Nierenversagen erlitten hatte. Auch diese Arbeit zeigte keine Unterlegenheit in der Funktion nach einem Jahr, wenn eine Niere mit ANV transplantiert wurde. Des Weiteren war das ANV in einer multivariaten Analyse kein Risikofaktor für die Einjahresfunktion.

\section{Klinische Relevanz}

Der Mangel an Spenderorganen führt immer häufiger zu einem „Sterben auf der Warteliste". Die Transplantation von Organen von Spendern mit einem akuten Nierenversagen bietet - bei Beachtung einiger Parameter wie normwertiges Kreatinin bei Aufnahme - eine Möglichkeit, den Spenderpool zu erweitern, ohne eine signifikante Verschlechterung der Funktionsraten in Kauf zu nehmen.

\section{Resistance-Index nach Nierentransplantation}

Der sonografisch ermittelte intrarenale Resistance-Index (RI) wird seit vielen Jahren als nichtinvasiver Marker nach Transplantationen eingesetzt, um einerseits die aktuelle Allograftfunktion zu beurteilen und andererseits die Prognose besser abschätzen zu können. Er setzt sich neben dem klassischen Gefäßwiderstand aus der Compliance der renalen Gefäße zusammen. In früheren Studien wurde ein renaler $\mathrm{RI} \geq 0,80$ mit einer Verschlechterung der Organfunktion assoziiert.

Einfluss auf Graft- und Patientenüberleben I Die Arbeitsgruppe um Naesens [16] untersuchte nun erneut, welchen Einfluss der RI auf die Graftfunktion sowie das Graft- und Patientenüberleben hat. Sie bestimmten prospektiv bei über 300 Patienten zu definierten Zeitpunkten sowie bei einer Indikations- oder Protokollbiopsie die Resistance im Bereich der Interlobararterien. Letztlich ergab diese große prospektive Studie keinen Zusammenhang des RI mit der Organfunktion, akuten oder chronischen Läsionen. Vielmehr zeigte sich eine Assoziation mit dem Empfängeralter sowie hämodynamischen Faktoren, d.h. der RI korrelierte mit dem Patienten- jedoch nicht mit dem Organüberleben. Der Cut-off betrug wie in den vorherigen Studien 0,80.

Vergleichbare Ergebnisse fanden sich bei der Korrelation der histologischen Ergebnisse der Protokoll- wie auch Indikationsbiopsien mit dem RI. Auch hier ist das Empfängeralter mit dem RI korreliert. Eine Ausnahme bildeten Biopsien, die aufgrund einer Graftdysfunktion durchgeführt wurden. Hier fand sich, zumindest in der univariaten Analyse, eine Korrelation mit einer akuten Tubulusnekrose sowie einer Antikörper-mediierten 


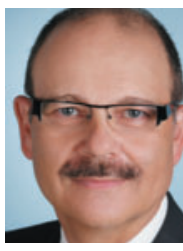

Prof. Dr. Werner Riegel ist Direktor der Medizinischen Klinik III, Klinikum Darmstadt GmbH w.riegel@

klinikum-darmstadt.de

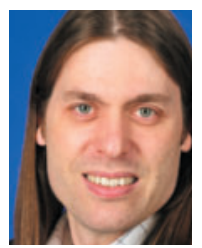

Prof. Dr. Bernd Krüger ist Leiter des Geschäftsbereichs Transplantation und Labor sowie Sprecher und nephrologischer Leiter des Transplantationszentrum Mannheim, V. Medizinische Klinik, Universitätsklinikum Mannheim bernd.krueger@umm.de

Interessenkonflikt

Die Autoren geben an, dass kein Interessenkonflikt besteht.

DOI 10.1055/s-0041-107694

Dtsch Med Wochenschr

2015; 140: 1831-1834

(c) Georg Thieme Verlag KG . Stuttgart · New York .

ISSN 0012-0472
Abstoßung. Nach einer Korrektur bzgl. multiplen Testens waren diese signifikanten Unterschiede jedoch nicht mehr vorhanden.

In einer weiteren Studie [17] wurde dieses Thema nochmals aufgenommen - mit einem Schwerpunkt auf kardiovaskulären Komplikationen. Die Autoren versuchten, der Spezifität des Spenderorgans durch eine paarweise Untersuchung Rechnung zu tragen. Sie identifizierten 266 Patienten die jeweils ein Organ desselben Spenders erhalten hatten und bei denen die RI-Messung zwischen dem 2. und 3. postoperativen Tag einen Unterschied von>0,1 ergab. In der Gruppe mit einem höheren RI kam es deutlich häufiger (54,1 vs. $20,3 \%, p<0,001)$ zu einer verzögerten Transplantatfunktion (DGF), definiert als Notwendigkeit einer Dialysebehandlung innerhalb der ersten Woche. Dieser Unterschied war nicht in einer höheren Rate an akuten Rejektionen oder durch supranormale Calcineurin-Inhibitorspiegel begründet. In den 3 Jahren nach Transplantation war die Nierenfunktion jedoch bei Patienten mit niedrigerem RI besser:

- 12 Monate: 47,6 vs. $53,2 \mathrm{ml} / \mathrm{min}(\mathrm{p}=0,02)$

- 24 Monate: 46,5 vs. $53,6 \mathrm{ml} / \mathrm{min}(\mathrm{p}=0,01)$

- 36 Monate: 46,2 vs. $52,1 \mathrm{ml} / \mathrm{min}(\mathrm{p}=0,03)$

Im Durchschnitt konnten die Patienten knapp über 9 Jahre nachbeobachtet werden, hier nivellierten sich diese Unterschiede aber zunehmend.

Des Weiteren berichten die Autoren von einer höheren kardiovaskulären Mortalität (11 vs. 3 Patienten) in der Gruppe mit einem höheren RI. Dies war in der univariaten Analyse signifikant, nicht mehr jedoch in der multivariaten Analyse, in die neben dem RI auch kardiovaskuläre Ereignisse vor Transplantation, Diabetes, DGF sowie das Empfängeralter eingingen. Letzteres war wieder mit dem RI assoziiert. Ferner fand sich in dieser Studie nach Durchführung einer „receiver operator curve“-Analyse (ROC), ein Resistance Index $>0,8$ als Diskriminator für das untersuchte Ereignis „kardiovaskuläre Mortalität“ (Sensitivität 73\%, Spezifität 47\%). Aufgrund der geringen Inzidenz der kardiovaskulären Mortalität ist die Aussagekraft dieser Studie eingeschränkt, bietet aber zusätzliche Argumente Patienten nach Transplantation in ihrem kardiovaskulären Risiko zu stratifizieren.

\section{Klinische Relevanz}

Der intrarenale Resistance-Index ist ein einfach zu erhebenden Marker zur Risikostratifizierung nach Nierentransplantation; seine Gesamt-Bedeutung ist weiterhin unklar.

\section{Literatur}

1 Weir MA, Dixon SN, Fleet JL et al. $\beta$-Blocker dialyzability and mortality in older patients receiving hemodialysis. J Am Soc Nephrol 2014; 26: 987-996

2 Ito Y, Mizuno M, Suzuki Y et al. Long-term effects of spironolactone in peritoneal dialysis patients. J Am Soc Nephrol 2014; 25: 1094-1102

3 Zhan L, Hao JB, Ren LS et al. The aldosterone receptor antagonist spironolactone prevents peritoneal inflammation and fibrosis. Lab Invest 2014; 94: 839-850

4 Vazquez-Rangel A, Soto V, Escalona $\mathrm{M}$ et al Spironolactone to prevent peritoneal fibrosis in peritoneal dialysis patients. 2014; 63: 1072-1074

5 Shapiro BB, Streja E, Ravel VA et al. Association of height with mortality in patients undergoing maintenance hemodialysis. Clin J Am Soc Nephrol 2015; 10: 965-974

6 Krummel T, Scheidt E, Borni-Duval $C$ et al. Haemodialysis in patients treated with oral anticoagulant: should we heparinize? Nephrol Dial Transpl 2014; 29: 906-913

7 Lavainne F, Meffray E, Pepper RJ et al. Heparin use during dialysis sessions induces an increase in the antiangiogenic factor soluble Flt1. Nephrol Dial Transpl 2014; 29: 1225-1231

8 Di Marco GS, Pavenstädt H, Brand M. Soluble Flt-1 release response to heparin use: implications for dialysis patients. Nephrol Dial Transpl 2014; 29: 1212-1215

9 US Food and Drug Administration (FDA). Eliquis (apixaban) Tablets. www.fda.gov/safety/medwatch/safetyinformation/ucm384790.htm

10 Contreras G, Pagan J, Chokshi R et al. Comparison of mortality of ESRD patients with lupus by initial dialysis modality. Clin J Am Soc Nephrol 2014; 9: 1949-1956

11 Guru P, Prakash R, Sheth $\mathrm{H}$ et al. Comparison of survival of patients with heart and lung transplants on peritoneal dialysis and hemodialysis. Perit Dial Int 2015; 35: 98-101

12 Paudel K, Fan SL. Successful use of continuous ambulatory peritoneal dialysis in 2 adults with gastrostomy. Am J Kidney Dis 2014; 64: 315-318

13 Farney AC, Rogers J, Orlando G et al. Evolving experience using kidneys from deceased donors with terminal acute kidney injury. J Am Coll Surg 2013; 216: 645-655

14 Benck U, Schnuelle P, Krüger B et al. Excellent graft and patient survival after renal transplantation from donors after brain death with acute kidney injury: a case-control study. Int Urol Nephrol 2015 Oct 26. [Epub ahead of print]

15 Jacobi J, Rebhan D, Heller K et al. Donor acute kidney injury and short-term graft outcome in renal transplantation. Clin Transplant 2014; 28 : 1131-1141

16 Naesens M, Heylen L, Lerut E et al. Intrarenal resistive index after renal transplantation. New Engl ] Med 2013; 369: 1797-1806

17 Kolonko A, Chudek J, Wiecek A. Initial kidney graft resistance index and the long-term cardiovascular mortality in transplanted patients: a paired grafts analysis. Nephrol Dial Transplant 2015; 30: 1218-1224 